\title{
A Study on the Framework Design of Artificial Intelligence Thinking for Artificial Intelligence Education
}

\author{
Seungki Shin
}

\begin{abstract}
This study aims to examine the definition and attributes of artificial intelligence (AI) thinking to support AI education, so educators can determine how such education should be conducted in grades $\mathrm{K}-12$. The text mining method was conducted using text crawling and co-word analysis to design and define AI thinking using the Python programming language. The cosine similarity and word 2 vec techniques were used to perform co-word analysis. Cosine similarity extracts paired words by assigning a weight according to the frequency of appearance. The skip-gram of word2Vec examines the surrounding words and predicts the paired words. According to the co-word analysis results, AI thinking is using an integrated thinking process to solve decision problems by discussing, providing, demonstrating, and proving processes. Moreover, AI thinking must be considered in future research on AI education. This study aims to serve as the foundational research to move forward in AI education.
\end{abstract}

Index Terms-Artificial intelligence (AI) thinking, co-word analysis, computer science, text crawling, cosine similarity, skip-gram.

\section{INTRODUCTION}

Artificial intelligence (AI) is expected to replace many societal roles and become the center of industry. These changes necessitate alterations to the content and direction of education to prepare people for the future by developing problem-solving abilities. The leading industrial countries, such as the United States, China, and South Korea, are preparing for a future with the education of an AI-based society [1]-[3]. Because education aims to enhance student skills required by society to prepare them for the future, the philosophy of supporting education and fundamental thinking skills is essential. Although considerable research on instructional methods for AI education exists, it is currently limited to explaining which thinking processes and philosophies are required for AI education [4], [5]. The study purpose is to examine the definition and attributes of $\mathrm{AI}$ thinking to support AI education so educators can determine how AI education should be conducted in grades $\mathrm{K}-12$.

\section{LITERATURE REVIEW}

\section{A. Artificial Intelligence and Education}

In 1956, John McCarthy proposed the first definition of AI at a Dartmouth conference: "An attempt will be made to find how to make machines use language, form abstractions and concepts, solve kinds of problems now reserved for humans,

Manuscript received January 7, 2021; revised May 27, 2021.

The author is with the Department of Computer Education, Seoul National University of Education, South Korea (e-mail: skshin@snue.ac.kr). and improve themselves" [6] (p. 2). Moreover, AI education is defined as the content and elements of teaching and learning that enhance creativity and problem-solving skills using AI technology and algorithms in computer science education. Further, AI education has three main educational benefits for K-12 students: (a) students learn the theory and principles of AI based on creativity [7], (b) students develop core skills required for the future [8], and (c) students enhance their communication skills and collaborative process by selecting effective algorithms to solve problems in automated work [9].

\section{B. Computational Thinking for Artificial Intelligence Education}

The recent concept of computational thinking was popularized by Wing [10]. To elaborate on the concept, she offered the following definition in 2010: "Computational thinking is the thought processes involved in formulating problems and their solutions so that the solutions are represented in a form that can be effectively carried out by an information-processing agent" [11] (para. 2). In addition, AI education is consistent with computational thinking in that decision-making uses algorithms based on logical reasoning, critical thinking, and analytical thinking [12]. This is based on the perspective that AI education should be part of computational thinking (e.g., [4], [12]-[14]).

Shin [14] presented an AI thinking model for CT-based AI education. The agency stage consists of the steps of data collection and the cognitive process to collect various data for problem-solving, check the data reliability, and solve the problem. The agency stage is designed to perform cognitive processes through cognitive reasoning, recursive reasoning, and repetition reasoning. The abstracting stage consists of discovering and categorizing the patterns of the collected data and defining the data using the common characteristics as a group. Finally, in the algorithm modeling stage, the algorithm is refined and generalized in the stages of modeling using trial and error and data visualization by applying the algorithm.

\section{RESEARCH METHOD}

This study aimed to design a framework of AI thinking for AI education. Using the Python programming language, the text mining method was conducted through text crawling and co-word analysis to design and define AI thinking. The text mining method is a series of steps to discover insight through text data extraction, data collection, and data analysis. Text mining has the advantage of finding meaningful insight by analyzing unstructured text data because more than $90 \%$ of the existing data are unstructured [15]. Python was employed to conduct text crawling and co-word analysis. 


\section{A. Text Mining Using Text Crawling}

The target data comprise the abstract information text from the Educational Advances in Artificial Intelligence (EAAI) conferences from 2016 to 2020. The EAAI conference is an educational section by the American Association of Artificial Intelligence, an authority in the field of AI industries and academia. The EAAI has a special interest in AI education and has been running for 10 years. To collect textual data on AI education, abstracts from submitted and presented full papers in the EAAI conference were collected using the text mining method using Python with text crawling libraries, such as Beautifulsoup. The Beautifulsoup library is a text crawling library that is effective and simple to conduct for Hypertext Markup Language (HTML) webpages using filtering with a parser of the HTML document with tags and attributes [16]. Because the papers presented at the EAAI conference are provided as HTML documents on the web, text crawling was performed using the Beautifulsoup library.

\section{B. Co-word Analysis Using Cosine Similarity}

Co-word analysis determines meaningful co-words in the context and document by extracting core words by comparing adjacent words [17]. Co-word analysis is divided into analyzing associations and assigning weights based on the frequency of appearance [17]. In this study, the latter method was used, and an analysis was performed by calculating the similarity between related words based on the frequency of appearance assigned with weights.

The weight was assigned using the term frequency (TF) inverse document frequency (IDF) method, and the similarity was calculated using the cosine similarity. The TF-IDF is a method of assigning a weight to the frequency of words by calculating the product of the TF (the frequency of word occurrence in a document) and the IDF (the value designating that the word commonly appears in the entire document group) [18], [19]. Equation (1) is a method for calculating $t f i d f$, and the $i d f$ value was calculated using the content of Equation (2) substituted into Equation (1). The following are the TF-IDF equations as follows [19]:

$$
\begin{aligned}
\operatorname{tfid} f(t, d, D) & =t f(t, d) \times i d f(t, D), \\
i d f(t, D) & =\log \frac{|D|}{|d \in D: t \in d|},
\end{aligned}
$$

$t f i d f(t, d, D)$ : Weight of the frequency as the product of $t f$ and $i d f$ values,

$t f(t, d)$ : Frequency of occurrence of the word $t$ in each document $d$,

$i d f(t, D)$ : Frequency of occurrence of word $t$ in entire document $D$,

$|D|$ : Total number of documents, and

$|d \in D: t \in d|$ : Number of documents containing the word $t$. If $t f(t, d)=0$, use the value of $1+|d \in D: t \in d|$.

Cosine similarity was used to examine the similarity between two words using the frequency of appearance of words assigned weights [20]. This was selected because it is a more widely used method than the Jaccard similarity and overlap similarity, which are other similarity calculation methods of text mining [16], [20]. The method of cosine similarity used to calculate the degree of similarity in this study is as shown in Equation (3) below, and Equation (4) was used to calculate the frequency of word appearance in the document as a vector value [16]. Cosine similarity equations are as follows [16], [20]:

$$
\begin{gathered}
\text { similarity }=\cos (\theta)=\frac{x_{i} \times x_{j}}{\left\|x_{i}\right\| \times|| x_{j} \|}, \\
S_{i j}=\sum_{k}\left(x_{i k} \times x_{j k}\right) / \sqrt{\left(\sum_{k} x_{i k}^{2}\right)\left(\sum_{k} x_{j k}^{2}\right)},
\end{gathered}
$$

$x$ : Frequency of occurrence of words,

$i, j$ : Two-word index for similarity analysis,

$k$ : Document index, and

$x_{i k}, x_{j k}$ : Vector values for $x_{i}$ and $x_{j}$, respectively.

\section{Co-word Analysis Using Word2vec}

Word2vec is an algorithm for co-word analysis, which was designed by Google [21]. Two assumptions are made to conduct the word2vec method for the meaning of a word [16]. First, the meaning of a word can be understood through the distribution of the words around the word [21]. Second, the meaning of a word can be encoded within word vectors so that the weight can be differentiated through the order of the words [21]. While the co-word analysis through cosine similarity is based on the frequency of word occurrence in the document, word2 vec handles the location, order of words, and frequency [22].

Two methods are used to conduct word2vec: the continuous bag-of-words (CBOW) and skip-gram [23]. The CBOW is a convergent analysis method that builds a model to predict a central word from the surrounding words [21], [23]. Skip-gram is a method of constructing a divergent model that predicts the surrounding words with a central word, which is the opposite direction of CBOW [22]. The skip-gram method was conducted in this study for co-word analysis through word2vec because the skip-gram method requires a greater learning process than the $\mathrm{CBOW}$ method, so it is more accurate [16], [22]. The equation of the skip-gram method for word2vec is as follows [22]:

$$
p\left(w_{c, j}=w_{O, c} \mid w_{I}\right)=\frac{\exp \left(u_{c, j}\right)}{\sum_{j^{\prime}=1}^{V} \exp \left(u_{j^{\prime}}\right)},
$$

$w_{c, j}$ : Predicted word through the $j^{\text {th }}$ order on the $c^{\text {th }}$ context, $w_{O, c}$ : Actual word in the $c^{\text {th }}$ context,

$w_{I}$ : Actual input word in the input vector, and

$u_{c, j}$ : Predicted word through the $j^{\text {th }}$ in output vector $U$ in the $c^{\text {th }}$ context.

Skip-gram is a form of unsupervised learning in machine learning that predicts output words based on an input word [16], [22]. As an algorithm of natural language processing (NLP), skip-gram is considered highly effective among recent NLP algorithms because it predicts the surrounding words by assigning weights through machine learning [16], [21], [22]. Because natural language has an atypical structure, it is effective to repeat it to obtain optimal results through learning rather than deriving results through one analysis [22], [23]. Therefore, this study derived a definition of AI thinking by comparing the results obtained through cosine similarity and the results from word2vec for the co-word analysis. 


\section{RESUlTS OF THE CO-WORD ANALYSIS}

\section{A. Co-word Analysis Using Cosine Similarity}

Abstracts of papers submitted to the EAAI conferences for the past five years were collected through data crawling to examine AI thinking characteristics. A total of 1,496 words were collected, and 107,807 co-words were extracted using the co-word analysis method. The extracted co-words were weighted using the TF-IDF method according to the frequency of occurrence of the words, and cosine similarity was applied to calculate the value of similarity between the co-words.

As listed in Table I, the top 20 paired co-words with the word "thinking" were listed as a result of cosine similarity. Based on the similarity results, the words were divided into four groups: Group 1 had a similarity value higher than 0.4. Group 2 consisted of words with a similarity value of between 0.3 and 0.4 . Words in Group 3 were selected with a similarity value of 0.2 but less than 0.3 , and Group 4 contained the remaining words. The extracted words determined using cosine similarity were indicated according to the similarity value using the word "thinking" and the number of occurrence frequencies in the documents.

The weight assigned by the TF-IDF is related to the frequency of occurrence between and within documents. In other words, the weight is low if the frequency of appearance between documents is high, and the weight is high if the frequency of appearance within the documents is high. Therefore, when the frequency of simultaneous occurrence of the paired words is high, but the degree of similarity is low, the paired words are mentioned in various documents. In contrast, if the frequency of the associated word's simultaneous occurrence is low and the similarity is high, the words are intensively presented in a specific document.

TABLE I: RESULTS OF CO-WORD ANALYSIS USING COSINE SIMILARITY

\begin{tabular}{|c|c|c|c|c|}
\hline Group & $\begin{array}{l}\text { Paired } \\
\text { Words }\end{array}$ & $\begin{array}{c}\text { Frequency } \\
\text { (a) }\end{array}$ & $\frac{\text { Similarity }}{\text { (b) }}$ & $\begin{array}{l}\text { Ratio of } \\
\text { (b) to (a) }\end{array}$ \\
\hline \multirow{10}{*}{ Group 1} & Example & 5 & 0.50 & 10 \\
\hline & Integrated & 3 & 0.49 & 16.3 \\
\hline & Decision & 5 & 0.48 & 9.6 \\
\hline & Complex & 5 & 0.47 & 9.4 \\
\hline & Problem & 17 & 0.45 & 2.6 \\
\hline & Develop & 21 & 0.44 & 2.1 \\
\hline & Computer & 9 & 0.43 & 4.8 \\
\hline & Students & 71 & 0.42 & 0.6 \\
\hline & Involved & 3 & 0.41 & 13.7 \\
\hline & Implications & 3 & 0.40 & 13.3 \\
\hline \multirow{3}{*}{ Group 2} & Approaches & 10 & 0.32 & 3.2 \\
\hline & Course & 34 & 0.31 & 0.9 \\
\hline & Module & 10 & 0.30 & 3 \\
\hline \multirow{4}{*}{ Group 3} & Science & 7 & 0.27 & 3.9 \\
\hline & Solution & 8 & 0.27 & 3.4 \\
\hline & Development & 9 & 0.23 & 2.4 \\
\hline & Algorithms & 20 & 0.20 & 1 \\
\hline \multirow{3}{*}{ Group 4} & Results & 22 & 0.17 & 0.8 \\
\hline & Projects & 7 & 0.17 & 2.4 \\
\hline & Challenge & 3 & 0.14 & 4.7 \\
\hline
\end{tabular}

Note: The ratio of the similarity to the frequency means the degree of similarity relevant to the word thinking compared to the number of words in the document. Paired words mean the words derived by calculating the cosine similarity with the word thinking to determine meaningful insight to define artificial intelligence thinking in education.

The ratio of the similarity to the frequency was also calculated as presented in Table I to examine the meaningful insight of the paired words.

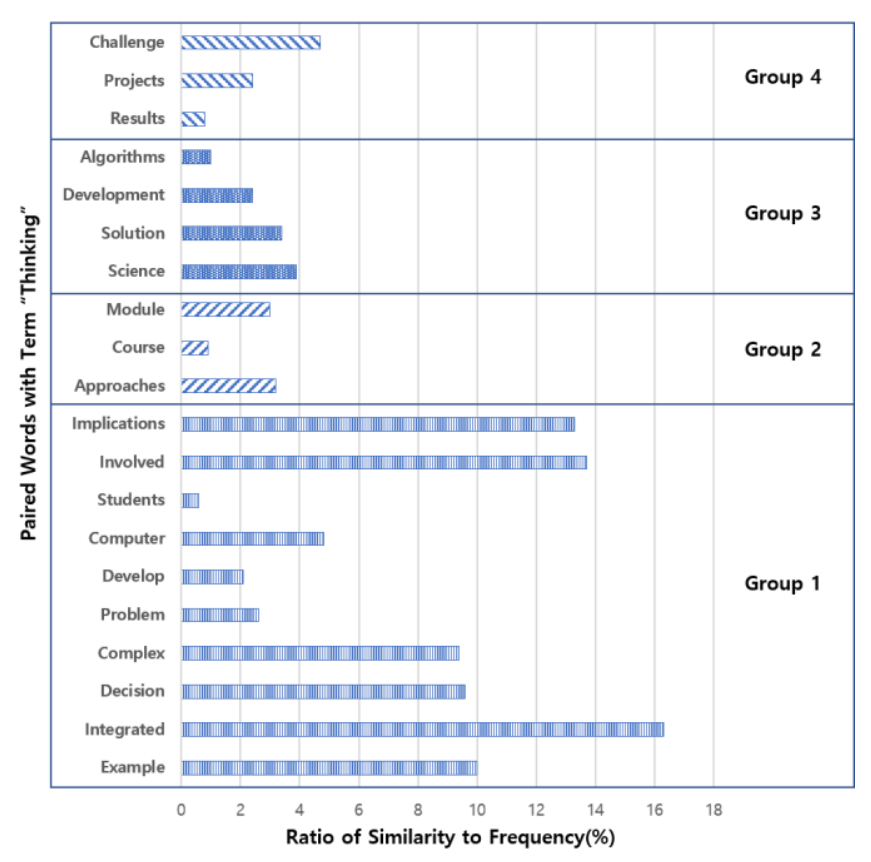

Fig. 1. Co-word analysis results by the similarity-to-frequency ratio.

According to the results of the ratio to the similarity of frequency in Fig. 1, the words "example," "integrated," "decision," "complex," "involved," and "implications" could be considered crucial to explain the definition of AI thinking. In other words, AI thinking could be defined as follows: AI thinking is the integrated thinking process to solve complex problems with decision-involved problems to determine the implications of the problems.

\section{B. Results of Co-word Analysis Using Word2vec}

Word2vec is an iteration-based method that creates a model to encode the probability of a word appearing in each context. It is a backpropagation method that evaluates errors while iterating the created model and then updates the model rules. It predicts the associated words by converting each word into a vector. The skip-gram algorithm was applied to perform word2vec, and the similarity was calculated by predicting the probability of distribution before and after the input word "thinking," as presented in Table II.

TABLE II: RESULTS OF CO-WORD ANALYSIS USING WORD2VEC

\begin{tabular}{llcc}
\hline \hline Input Word & Output Words & Frequency & Similarity \\
\hline Thinking & Prove & 15 & 0.31 \\
& Conversations & 6 & 0.28 \\
& Decision & 5 & 0.27 \\
& Discuss & 10 & 0.24 \\
& Providing & 5 & 0.22 \\
Analytic & 8 & 0.22 \\
& Technique & 8 & 0.22 \\
& Demonstrate & 7 & 0.22 \\
& Answer & 10 & 0.21 \\
& Development & 9 & 0.21
\end{tabular}

Note: The skip-gram method was employed with the word2vec algorithm The word "thinking" was set as the central word, and up to two words on the left and right that appeared at least twice in the entire document were learned using unsupervised learning.

Co-word analysis was performed by calculating the 
similarity of two words derived more than once in the same context before and after the input word "thinking" because the study purpose was to examine AI thinking characteristics to define the AI thinking process. The top 10 words for the predicted similarity results are listed in Table II with the frequency of appearance in the entire document. Because the frequency of occurrence of a word is the number of times it is mentioned in the entire document, it can be judged as a keyword in AI education research. The predicted similarity results are the words expressing the characteristic of AI thinking in that they indicate relevance to the term "thinking."

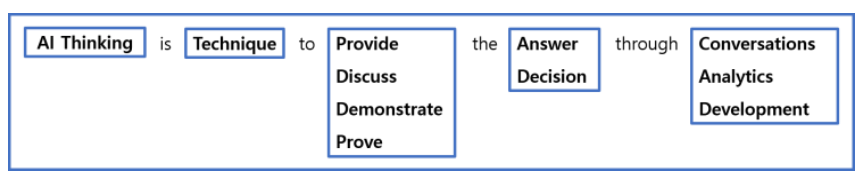

Fig. 2. Organized results of the co-word analysis using skip-gram with word2vec

As displayed in Fig. 2, the extracted words using the skip-gram method of word2vec were reorganized to construct a sentence to explain AI thinking characteristics. The words "provide," "discuss," "demonstrate," and "prove" can be considered verbs to conduct AI thinking to determine solutions by finding the "answer" or "decision" concerning the problem. Meanwhile, AI thinking can be processed through the following activities: "conversations," "analytics," and "development." These verbs, activities, and expected results to conduct AI thinking can be defined as a "technique" in a series of thinking processes to solve a problem.

\section{FRAMEWORK OF AI THINKING}

Additionally, AI thinking is a cognitive process performed for problem-solving in AI education. Education is a series of processes in which instructional methods are applied based on learner-centered learning theory. In other words, AI thinking can be considered a type of learning to conduct AI education. Reigeluth and Moore's synthesis of taxonomies for learning types was applied to design AI learning for AI thinking comprising memorizing information, understanding relationships, and applying specific and generic skills to design the framework of AI thinking in this study [24].

\begin{tabular}{|c|c|c|}
\hline Memorize Information & & Apply Skills \\
\hline & & Science \\
\hline Approaches & 1 & Solution \\
\hline Course & I & 10 \\
\hline Module & $\begin{array}{l}\text { I } \\
\text { I }\end{array}$ & Algorithms \\
\hline Example-Decision & I & $\begin{array}{l}----------- \\
\text { Results }\end{array}$ \\
\hline Problem-Implications & $\mathbf{I}$ & \\
\hline Develop-Involved & I & projects \\
\hline Computer-Students & I & Challenge \\
\hline Integrated-Complex & & \\
\hline Understand Relationships & & Apply Generic Skills \\
\hline
\end{tabular}

Fig. 3. Type of artificial intelligence (AI) learning for AI thinking.

Based on the results of the co-word analysis using cosine similarity, each group was assigned to a related type of learning, as illustrated in Fig. 3. Memorizing information is about understanding the relevant knowledge necessary for learning; thus, Group 2 was assigned, which is related to the technical understanding of AI. Understanding relationships is a process to understand problems based on prior knowledge and discover what problems need to be solved. Group 1 is assigned to understanding relationships because it comprises words for the problem-solving process and creates an environment to solve problems through AI. Applying skills is the step in which problem-solving ideas are designed, and a series of problem-solving processes are conducted. Group 3 was assigned to the apply skills category, which is the process of developing scientific solutions and implementing them with algorithms. Applying generic skills was assigned to Group 4 because it refers to generalizing problem-solving results to other problems through new challenging problems, reviewing the results of the problem-solving process, and generalizing problem-solving skills using AI.

We use AI in the real world because AI helps solve problems using automated computing processes through algorithms. In other words, a benefit of AI is solving problems as a technique using computational processes following the human cognitive thinking process. Based on the co-word analysis results using skip-gram, which predicted words related to thinking, the extracted words were reorganized to explain AI thinking characteristics, as illustrated in Fig. 4.

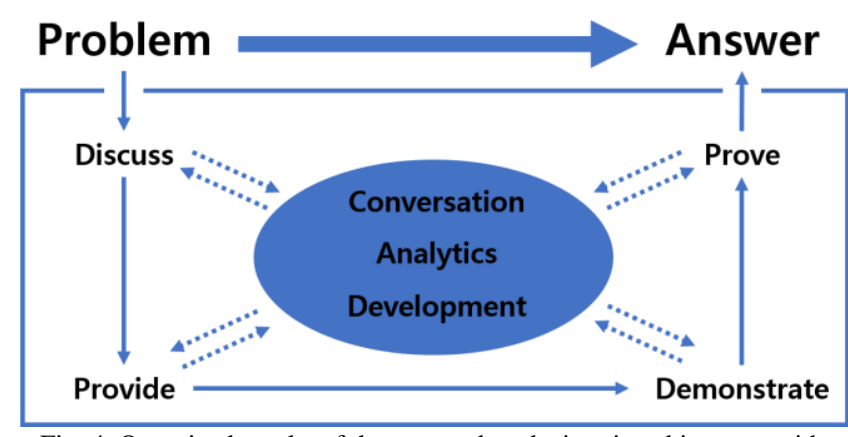

Fig. 4. Organized results of the co-word analysis using skip-gram with word 2 vec.

In the figure, the outside of the box is the external thinking process, meaning the process of finding and solving problems, and the inside of the box is the intrinsic thinking process, meaning a complex cognitive thinking process performed to solve the problem. The first step is to conduct a discussion with others or use prior internal knowledge to discover the problem. The next step is to provide various ideas for problem-solving. Discovered ideas are checked to determine whether they are appropriate for problem-solving through demonstration. The appropriated idea is used in the problem-solving process using AI to prove its effectiveness. In this series of AI thinking steps, the conversation with others or inner knowledge, analytics of the problem and solution, and development of the problem-solving process are repeatedly used as needed in performing the internal thinking process. In other words, AI thinking can be expressed as an inner series of thinking processes using AI to find problems and answers.

If the AI thinking framework in Fig. 4 is applied to the educational field, it can be divided into teacher and student activities. Teachers provide scaffolding for the external thinking process and present problem situations as facilitators. 
Problem situations should be solved by students by discussing and discovering, explaining the problem to be solved, and applying AI technology. For example, teachers could introduce phenomena caused by climate change. Students could predict the future climate by analyzing the existing data on precipitation and the average temperature and discover problems.

Students should discuss topics to provide ideas for solving the discovered problems and should be able to analyze and develop predictable problem-solving methods using AI technologies, such as machine learning. Teachers should help students with the difficulties in AI technology. However, they should not have a decisive influence on student decision-making and should play a facilitator role. For example, when an idea for solving climate change is applied, the effect on precipitation and the average temperature is predicted through linear regression analysis and is continuously demonstrated to determine the most effective problem-solving ideas.

Students select the most appropriate problem-solving ideas and prove them through the process of presenting and discussing them. It is the final step in the series of processes of discovering and solving problems. The idea of problem-solving can be continuously updated, and new problems can be discovered and solved through iteration. Suppose, for example, that an AI technology is applied to address climate change to present the plans and expected results to lower the carbon dioxide generation rate from automobiles and factories. In a presentation, new problems can be discovered through questions from and discussions with classmates or teachers. To lower automobile operation, public transportation or an eco-friendly means of transportation can be employed. In repetitive problem-solving, students perform problem-solving and apply AI technology. Creative problem-solving skills can be expected to increase through human cognitive thinking processes and AI automation technology.

\section{CONCLUSION AND SUGGESTIONS}

Education is critical in the age of AI [25]. Because AI is an automated computing algorithm that mimics human cognitive intelligence, the importance of education is emphasized to cultivate primary skills. Thus, education to cultivate thinking skills for problem-solving is vital and emphasizes the need to learn about thinking processes using AI along with understanding AI technology. Kaplan stated, "An attempt will be made to find how to make machines use language, form abstractions and concepts, solve kinds of problems now reserved for humans, and improve themselves" [25] (p. 13).

It is essential for students living in the era of the fourth industrial revolution to learn AI technology. Countries are pushing ahead with attempts to introduce AI in education to cultivate learners' future capabilities. About 70 years have passed since the definition of AI technology was introduced, but interest in $\mathrm{AI}$ has recently increased with the development of information and communication technology. Considerable research on the content and learning materials for AI education exists, but research on pedagogical approaches is currently insufficient. Because AI education is based on programming, it should be approached as part of computer science education. Computer science education is based on computational thinking, so AI education should take the same direction. However, AI technology imitates the human learning process through machine learning; thus, AI thinking focused on the thinking process must be carried out for AI education.

In this study, a co-word analysis was performed through NLP technology using AI technology to define AI thinking and examine the procedures and characteristics. Cosine similarity and word2vec techniques were used to perform the co-word analysis. Cosine similarity extracts paired words by assigning a weight according to the frequency of appearance, and the skip-gram with word2vec examines the surrounding words and predicts the paired words. Through co-word analysis using cosine similarity, AI thinking is an integrated thinking process to solve decision-involved problems. According to the co-word analysis results using skip-gram with word2vec, we examined the processes and properties of AI thinking and found that the processes of discussing, providing ideas, and demonstrating answers to prove answers are needed to solve problems. Moreover, AI thinking must be considered in future research on AI education, and this study can serve as foundational research to move forward in AI education.

\section{CONFLICT OF INTEREST}

The author declares no conflict of interest.

\section{REFERENCES}

[1] D. Touretzky, F. Martin, D. Seehorn, C. Breazeal, and T. Posner, "Special session: AI for K-12 guidelines initiative," in Proc. the 50th ACM Technical Symposium on Computer Science Education, pp. 492-493, 2019.

[2] (2017). Next-generation artificial intelligence development plan. China Government. [Online]. Available: http://www.gov.cn/zhengce/content/2017-07/20/content_5211996.htm

[3] (2020). Educational policy and core tasks in the age of artificial intelligence. Government of the Republic of Korea. [Online]. Available:

http://www.moe.go.kr/boardCnts/fileDown.do?fileSeq=29125bbe5a1f 9 bce 117ba72cfc0e481e

[4] S. Shin, "Designing the instructional framework and cognitive learning environment for artificial intelligence education through computational thinking," Journal of the Korean Association of Information Education, vol. 23, no. 6, pp. 639-653, December 2019.

[5] S. Shin, "Designing the framework of evaluation on learner's cognitive skill for artificial intelligence education through computational thinking," Journal of the Korean Association of Information Education, vol. 24, no. 1, pp. 59-69, February 2020.

[6] J. McCarthy, M. L. Minsky, N. Rochester, and C. E. Shannon, "A proposal for the dartmouth summer research project on artificial intelligence," AI Magazine, vol. 27. no. 4, pp. 12, August 1955.

[7] S. Ree and Y. Koh, "The aims of education in the era of AI," Journal for History of Mathematics, vol. 30, no. 6, pp. 341-351, December 2017.

[8] J. Park and N. Shin, "Students' perceptions of artificial intelligence technology and artificial intelligence teachers," The Journal of Korean Teacher Education, vol. 34, no. 2, pp. 169-192, June 2017.

[9] M. R. K. Krishna Rao, "Infusing critical thinking skills into content of AI course," in Proc. the 10th Annual SIGCSE Conference on Innovation and Technology in Computer Science Education, pp. 173-177, vol. 37, no. 3, June 2005.

[10] J. Wing, "Computational thinking," Communications of the ACM, vol. 49, no. 3, pp. 33-35, March 2006.

[11] J. Wing, "Research notebook: Computational thinking — What and why?" The Link Magazine, Spring. Pittsburgh, PA: Carnegie Mellon University, 2011.

[12] P. Silapachote and A. Srisuphab, "Engineering courses on computational thinking through solving problems in artificial 
intelligence," International Journal of Engineering Pedagogy, vol. 7, no. 3, pp. 34-49, September 2017.

[13] G. Gadanidis, "Artificial intelligence, computational thinking, and mathematics education," The International Journal of Information and Learning Technology, vol. 34, no. 2, pp. 133-139, March 2017.

[14] W. C. Shih, "Integrating computational thinking into the process of learning artificial intelligence," in Proc. the 2019 3rd International Conference on Education and Multimedia Technology of ACM, pp. 364-368, July 2019.

[15] S. M. Weiss, N. Indurkhya, T. Zhang, and F. Damerau, Text Mining: Predictive Methods for Analyzing Unstructured Information, 1st ed. New York, NY: Springer Science \& Business Media, 2010.

[16] D. Seo, Catch it! Text Mining with Python, 1st ed. Seoul, South Korea: BJPublic, 2019.

[17] B. Delecroix and R. Epstein, R. "Co-word analysis for the non-scientific information example of Reuters Business Briefings," Data Science Journal, vol. 3, pp. 80-87, January 2006.

[18] A. Aizawa, "An information-theoretic perspective of TF-IDF measures," Information Processing \& Management, vol. 39, no. 1, pp. 45-65, January 2003.

[19] J. Ramos, "Using TF-IDF to determine word relevance in document queries," in Proc. the First Instructional Conference on Machine Learning, vol. 242, pp. 133-142, December 2003.

[20] National Institute of Standards and Technology. (July 2017). Cosine distance, cosine similarity, angular cosine distance, angular cosine similarity. Dataplot. Statistical Engineering Division. [Online]. Available:

https://www.itl.nist.gov/div898/software/dataplot/refman2/auxillar/co sdist.htm

[21] T. Mikolov, I. Sutskever, K. Chen, G. S. Corrado, and J. Dean, "Distributed representations of words and phrases and their compositionality," Advances in Neural Information Processing Systems, vol. 26, pp. 3111-3119, October 2013.

[22] X. Rong. (November 2014). Word2vec parameter learning explained. [Online]. Available: https://arxiv.org/abs/1411.2738

[23] T. Mikolov, K. Chen, G. Corrado, and J. Dean. (January 2013). Efficient estimation of word representations in vector space. [Online]. Available: https://arxiv.org/abs/1301.3781

[24] C. M. Reigeluth and J. Moore, "Cognitive Education and the cognitive domain," Instructional-Design Theories and Models: A New Paradigm of Instructional Theory Volume II, Mahwah, NJ: Lawrence Erlbaum Associates, 1999, pp. 51-68.

[25] J. Kaplan, Artificial Intelligence: What Everyone Needs to Know, New York, NY: Oxford University Press, 2016.

Copyright $\odot 2021$ by the authors. This is an open access article distributed under the Creative Commons Attribution License which permits unrestricted use, distribution, and reproduction in any medium, provided the original work is properly cited (CC BY 4.0).

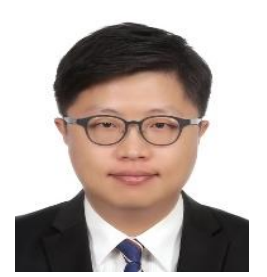

Seungki Shin was born in Pohang, South Korea in 1983. He has received a bachelor of education degree with a double major in computer science education and elementary education at Korea National University of Education, Cheongju, South Korea in 2007. He received the M.Eng. degree in information and communication technology from Ajou University, Suwon, South Korea in 2009. He earned a master of education degree in computer education at Daegu National University of Education, Daegu, South Korea in 2012. He has received a $\mathrm{Ph} . \mathrm{D}$. degree in learning, design, and technology from the Department of Career and Information Studies at the University of Georgia, Athens, GA, USA in 2017. His major field of study is computer science education.

He was a graduate researcher at Carl Vinson Institute of Government at the University of Georgia from 2016 to 2017, a lecturer with the Department of Computer Education at Daegu National University of Education, South Korea from 2017 to 2019, and a clinical assistant professor of Computer Science Education with Division of Teacher Preparation and affiliated faculty member with Center for Advanced Studies in Global Education in Mary Lou Fulton Teachers College at Arizona State University from 2019 to 2020. He is now an assistant professor with the Department of Computer Education, Seoul National University of Education, Seoul, South Korea. The current research interests of him are computer science education, computational thinking, educational programming language, physical computing education, and artificial intelligence education.

Dr. Shin is a border member of the Korean Association of Information Education and a member of the Computer Science Teachers Association. He has published lots of articles on curriculum development for computer science education and studies on instructional design and model development for computational thinking 